\title{
Medida de atitudes em contextos organizacionais masculinos
}

\author{
Rosa Monteiro ${ }^{1}$ \\ Amélia Filipa Soares Vieira ${ }^{2}$ \\ Fernanda Daniel ${ }^{12}$ \\ Alexandre Gomes da Silva ${ }^{13}$ \\ Fátima Regina Ney Matos ${ }^{2}$ \\ ${ }^{1}$ Universidade de Coimbra, Coimbra, Portugal \\ ${ }^{2}$ Instituto Superior Miguel Torga, Coimbra, Portugal \\ ${ }^{3}$ Instituto Superior de Contabilidade e Administração de Coimbra / Coimbra Business School, Coimbra, Portugal
}

Compreender a persistência de concepções assimétricas assentes em estereotipia de gênero de trabalhadores é fundamental para as organizações que desejem desenvolver planos de gestão para a igualdade. O propósito deste estudo, realizado em Portugal, foi identificar se em contextos organizacionais altamente masculinizados, ou seja, majoritariamente masculinos, a estereotipia de gênero tende a ser mais acentuada. Para sua realização, aplicou-se o questionário "Men's Polarized Gender Thinking (MPGQ)" a uma amostra de 160 trabalhadores de 2 organizações Águas Limpas e SOS. As participações no mercado de trabalho dos homens com níveis de instrução mais elevados se articulam com atitudes mais igualitaristas, ao passo que são os menos escolarizados que menos reconhecem as desigualdades. A escolarização e a educação dos indivíduos são consideradas fundamentais, pois se destacam como importante vetor de aquisição de um sistema de crenças e de valores sociais mais igualitários. Os resultados. Os resultados obtidos demonstram que as atitudes dos homens em relação à igualdade de gênero não se distribuem aleatoriamente. Esses achados corroboram alertas lançados pelas abordagens da interseccionalidade.

Palavras-chave: segregação horizontal; (des)igualdades; gênero.

\section{Medición de actitudes en contextos organizacionales masculinos}

Para entender la persistencia de concepciones asimétricas basadas en las estereotipias de género de los trabajadores, es fundamental que las organizaciones deseen desarrollar planes de gestión para la igualdad. El propósito de este estudio, realizado en Portugal, fue identificar si en contextos organizacionales altamente masculinizados la estereotipia de género tiende a ser más acentuada. Para su realización se aplicó el cuestionario Men’s Polarized Gender Thinking en una muestra de 160 trabajadores de dos organizaciones: Águas Limpas y SOS. La participación en el mercado de trabajo de los hombres con niveles más elevados de instrucción está vinculada con actitudes más igualitarias, mientras que los menos escolarizados son los que menos reconocen las desigualdades. La escolarización y educación de los individuos se consideran fundamentales, ya que se destacan como un vector importante para la adquisición de un sistema de creencias y valores sociales más igualitario sobre el tema de las relaciones de género. Los resultados obtenidos muestran que las actitudes de los hombres hacia la igualdad de género no se distribuyen aleatoriamente. Estos hallazgos corroboran las advertencias emitidas por los enfoques de interseccionalidad.

Palabras clave: segregación horizontal; (des)igualdades; género.

\section{Measurement of attitudes in masculine organizational contexts}

Organizations developing equality plans must understand the persistence of workers' asymmetric conceptions based on gender stereotype. This study was conducted in Portugal and aimed to identify whether there are more gender stereotypes in highly masculinized organizational contexts. A questionnaire "Men's Polarized Gender Thinking Questionnaire (MPGQ)" was applied to a sample of 160 workers from two organizations: Águas Limpas and SOS. In the labor market, men with higher levels of education tend to present more equalitarian attitudes, while less educated men are less likely to recognize inequalities. Individuals' schooling and education are crucial, as these aspects stand out as important vectors for the acquisition of a more equalitarian system of beliefs and social values on the theme of gender relations. The results show that the attitudes of men towards gender equality are not distributed randomly. These findings corroborate the warningsmade in research adopting intersectionality approaches.

Keywords: horizontal segregation; (un)equalities; gender. 


\section{INTRODUÇÃO}

Em Portugal, não obstante os progressos formais e legais em matéria de igualdade de mulheres e homens no trabalho e no emprego (Monteiro, 2010), na realidade, são inúmeras as desigualdades e mesmo as discriminações em razão do sexo que subsistem (Monteiro \& Ferreira, 2013; Ornelas, Matos, Machado, \& Mesquita, 2017). Segundo Nogueira (2001), o que persiste, no entanto, é a distância entre a igualdade legal formal e política e a prática de todos os dias. Os direitos e os princípios se mantiveram nos planos teóricos, especialmente em termos socioeconômicos e no que diz respeito às vidas privadas das mulheres.

Assim, as esferas do trabalho (pago e não pago) e do emprego constituem espaços ideais de observação e de transformação das relações sociais de gênero, em suas consequências nefastas e seus resultados assimétricos para uns e para outras. "Gênero" é o termo proposto por Ann Oakley para se referir ao fato de, sobre as diferenças biológicas entre os dois sexos, construírem-se social e culturalmente significados e pressupostos sobre "feminino" e "masculino", sobre "ser mulher" e "ser homem", que se impõem nas cognições sociais e individuais, produzindo e reproduzindo estereotipias de gênero (Amâncio, 1992; Monteiro, 2005).

Os estereótipos de gênero são opiniões ou preconceitos generalizados sobre atributos ou características que homens e mulheres possuem ou deveriam possuir ou as funções sociais que desempenham ou deveriam desempenhar constituem entraves simbólicos, com impactos nas práticas organizacionais e de gestão, condicionando decisões e atribuições em função de uma ideia estereotipada de masculinidade e de feminilidade.

Sealy e Singh (2010) defendem que as culturas organizacionais são altamente resilientes às concepções de gênero favoráveis ao poder masculino, resistindo veementemente a iniciativas de promoção da igualdade de oportunidades. Broadbridge e Simpson (2011), por seu turno, entendem que as hierarquias de gênero estão a se tornar mais fortes, facilitadas por discursos de igualdade feminina e pela primazia dada à meritocracia e à escolha, retóricas particularmente bem acolhidas entre as gerações mais jovens de gestores/as. As mesmas autoras alertam, porém, para um conjunto de fatores que invisibilizam essas desigualdades e assimetrias que importa combater.

Como efeito, nas organizações de trabalho e nos estudos de gestão, a dimensão de gênero tem sido escondida, invisibilizada por uma ideologia de gestão neutra e de irrelevância diante das conquistas já alcançadas. Como referem Broadbridge e Simpson (2011), o gênero tem sido historicamente ignorado ou marginalizado na pesquisa mainstream em gestão, ainda que crescentemente integrada nos domínios da gestão de recursos humanos, comportamento organizacional e liderança (negligenciado nos estudos nas áreas de marketing, finanças e produção) (Broadbridge \& Hearn, 2008). Isso acontece por três principais ordens de razões: porque se entende que as questões da desigualdade entre mulheres e homens estão “já resolvidas” e a igualdade já é uma realidade (deduzida, muitas vezes, da falsa evidência de que as mulheres "já estão por todo lado"); porque predomina hoje uma atenção à gestão da diversidade que diluí a problemática específica da (des)igualdade de gênero; pelo poderoso discurso da meritocracia e da escolha pessoal que atualmente prevalece no domínio da gestão, com influência especial nas mais novas gerações de gestores/as. 
Esse discurso leva a reinterpretar as desvantagens de gênero, em especial as das mulheres, não como uma questão estrutural, institucional e cultural, mas como uma questão de escolhas pessoais (Broadbridge \& Simpson, 2011). Ainda relativamente ao primeiro motivo, o enaltecimento de "qualidades femininas" nas novas abordagens de gestão, que destacam a importância das emoções, do trabalho em equipe e da comunicação, bem como os programas para a capacitação das mulheres para a chefia e liderança, criaram a visão de que "já está tudo feito" e de que já existe igualdade.

Diante disso, tem sido sublinhada, na literatura (Agência Europeia para a Segurança e Saúde no Trabalho, 2013; Broadbridge \& Simpson, 2011; Fagan \& Burchell, 2002), a necessidade de visibilizar e conceitualizar as desigualdades em razão do sexo, mas subestudadas, no universo do trabalho e das organizações, expressas não apenas nas práticas de gestão e nos resultados alcançados por homens e mulheres, mas também em dimensões simbólicas, culturais e identitárias da vida organizacional que influenciam aquelas práticas e resultados. Por essa razão, uma das estratégias de investigação propostas por Broadbridge e Simpson (2011) se destina a desvendar os aspectos escondidos e a ocultação das questões de gênero nas normas, práticas e valores presentes nas organizações e nos sujeitos organizacionais, além de perceber como as identidades genderizadas são construídas e mantidas em diferentes contextos organizacionais.

Esse foi, precisamente, o principal propósito deste estudo: partindo da observação de um contexto organizacional altamente masculinizado, buscou-se identificar se, em universos profissionais muito segregados, a estereotipia de gênero tende a ser mais acentuada. Além das concepções sociais de gênero penosas para as mulheres e para a sua afirmação profissional, ambientes hostis à igualdade acarretam custos para as organizações por excluírem competências e capital humano. Como demonstrou o estudo "The Athena Factor" (Hewlett et al., 2008), no setor americano da ciência, novas tecnologias e engenharias, culturas de trabalho e organizacionais masculinizadas representam ambientes hostis que excluem e afastam mulheres profissionais, em um fenômeno de braindrain fenômeno que se refere à perda de capital/recursos humanos com níveis de formação elevados. Tal fenômeno é reportado na literatura em associação à saída de profissionais com níveis de formação superior de países menos desenvolvidos ou hostis para países mais desenvolvidos; no contexto deste estudo, o fenômeno é estendido à perda de capital/recursos humanos femininos - penalizador para o próprio setor, especialmente na iniciativa privada. Um ambiente masculinizado é aquele onde os homens têm maioria numérica, constatando-se a segregação horizontal pela prevalência de tarefas estereotipadamente consideradas masculinas.

Outros estudos, no âmbito da gestão, consideram importante conhecer as crenças e atitudes de gênero dos homens trabalhadores, como forma de potenciar a entrada de mulheres em setores tradicionalmente masculinizados e de alterar aspectos de uma cultura e estrutura masculina nos locais de trabalho evitando a discriminação e desigualdade, sobretudo das mulheres (Bergman, Larsman, \& Löve, 2014; Smith, Crittenden, \& Caputi, 2012).

Após esta introdução, na primeira parte, apresenta-se o enquadramento teórico, explicitandose a linha analítica que orienta o estudo, as concepções e conceitualizações fundamentais para a análise da problemática; na segunda parte, expõe-se a metodologia, a caracterização da amostra, os instrumentos de coleta de dados e as técnicas de análise de dados; por fim, na terceira parte, apresentase a discussão/conclusão. 


\section{ORGANIZAÇÕES E GÊNERO: SEGREGAÇÃO HORIZONTAL E CULTURAS ORGANIZACIONAIS HOSTIS À IGUALDADE}

Depois de décadas de vigência da ideia de uma suposta neutralidade da gestão e das organizações, tem-se demonstrado que as relações sociais de gênero são criadas e recriadas na esfera do trabalho, não só pela ação das pessoas, mas também pela ação das organizações (Acker, 1990, 2009; Collinson, 1992; Fenstermaker, West, \& Zimmerman, 1991; Kanter, 1993; Morgan, 1992; Reskin \& Padavic, 1994).

Fala-se em genderização das organizações, processo definido como as construções materiais e discursivas da masculinidade e da feminilidade que moldam e são moldadas pelos sistemas, práticas de trabalho, normas e identidades das organizações (Benschop \& Verloo, 2006).

Acker (2009) forneceu o contributo mais destacado para esse reconhecimento, ao defender que todas as organizações têm "regimes de desigualdade" definidos como práticas, processos, ações e significados interrelacionados que mantêm as desigualdades de classe, de gênero e raciais dentro das organizações.

Acker (1992) nos apresenta quatro níveis de manifestação desses processos de genderização das organizações. A primeira corresponde à produção de divisões de gênero nas estruturas organizacionais. Esta se expressa em fenômenos de segregação, como, por exemplo, a reduzida presença de mulheres em cargos de decisão (designada como segregação vertical, ou glassceiling), ou na existência de profissões predominantemente ocupadas por um dos sexos (segregação horizontal, ou glasswalls). Embora desafiadas pelas abordagens interseccionais inauguradas por Crenshaw (1989), que contestam a suposição de um sujeito unitário e homogêneo subjacente aos feminismos liberais, esses conceitos permanecem com forte poder heurístico quando se trata de analisar processos culturais como aqueles subjacentes às concepções de gênero produzidas e reproduzidas pelos sujeitos e nas organizações. $\mathrm{O}$ segundo nível diz respeito à presença de representações simbólicas de masculinidade e feminilidade nas culturas organizacionais, que se expressa de forma mais ou menos latente. Os estereótipos de gênero constituem entraves simbólicos, com impactos nas práticas organizacionais e de gestão, condicionando decisões e atribuições em função de uma ideia estereotipada de masculinidade e de feminilidade. Um desses impactos é bem visível, por exemplo, nos entraves aos direitos de paternidade por parte dos trabalhadores do sexo masculino, como comprovou Lopes (2009). Outro nível é o que corresponde aos processos de interação social que marcam a vida das organizações e dos homens e mulheres que nelas trabalham. Com efeito, e como demonstraram diversos estudos, a interação social nas organizações de trabalho tem uma marca de genero (Monteiro, 1995; Reskin \& Padavic, 1994). Finalmente, o quarto nível, mais de microanálise, mas extremamente relevante, é o que se situa no estrato do sujeito organizacional e da sua identidade como pessoa sexualizada, carregando experiências, concepções e práticas produtoras e reprodutoras das relações sociais de gênero. Fenstermaker et al. (1991) designaram esse processo como doing gender.

A importância das cognições e representações de gênero dos trabalhadores nas organizações também chega até nós por Fagenson (1990). A autora entende que os comportamentos das pessoas nas organizações de trabalho são genderizados, ou seja, produtos e produtores de concepções de gênero, e isso determina suas racionalidades, seus pensamentos, suas opções e suas práticas. Assim, e globalmente, segundo a autora, o comportamento humano nas organizações deve ser estudado como produto articulado das características individuais, da situação (cultura organizacional) e do sistema institucional e social mais global, todas elas determinadas pelas concepções de gênero. Tais 
concepções condicionam a forma como as pessoas se representam e como representam os outros nos diversos contextos e esferas de vida, mas também as práticas concretas de e no trabalho que adotam, por exemplo, por isso devem ser consideradas pela gestão.

A segregação horizontal, conceito que ilustra o fato de estereotipadamente se definirem profissões mais adequados para um dos sexos, tendo como consequência sua concentração profissional e setorial, é uma das consequências mais visíveis das concepções de gênero nas organizações e no trabalho.

Estudos têm demonstrado o especial impacto da segregação ocupacional na determinação de ambientes sexistas e hostis à igualdade entre mulheres e homens. Hewlett et al. (2008), elaboraram um estudo, financiado por cinco grandes multinacionais norteamericanas (Alcoa, Cisco, Johnson \& Johnson, Microsoft e Pfizer), no sentido de compreender o brain drain no setor da ciência, engenharias e tecnologia, nos Estados Unidos da América (EUA). O relatório, publicado pelo Center for Work-Life Policy, de Nova York e intitulado The Athena Factor: reversing the brain drain in science, engineering, and technology (Hewlett et al., 2008), parte da constatação do problema de escassez de trabalhadores/as qualificados/as nas empresas do setor científico, tecnológico e das engenharias, por cerca de 52\% das mulheres, com idades entre 35 e 40 anos, estarem a abandonar seus empregos no setor devido a "ambientes de trabalho hostis e às fortes pressões profissionais".

Segundo o relatório, a cultura machista exclui as mulheres dos espaços de decisão - os after hours nos laboratórios, nos bares ou nos campos de golfe -, privando as mulheres de informação vital. A exclusão não é apenas física, atuando também via comportamentos depreciativos e humilhantes e linguagem rude, vulgar e até sexualmente explícita, que isolam ainda mais as mulheres nesses ambientes (63\% descreveram situações de assédio sexual). Segundo as mulheres inquiridas, tais comportamentos são desculpados e até mimetizados pela própria liderança das empresas. Como uma das entrevistadas confessou, eis a resposta que obteve da direção de recursos humanos depois de queixar-se dessas situações: "assim não vais conseguir ter sucesso; tens de te esforçar mais para te tornares um dos rapazes" (Hewlett et al., 2008, p. 7).

São categorizados cinco grandes motivos para essa saída das mulheres do setor: as culturas masculinas hostis; o isolamento das mulheres e a falta de apoio e de mentoria; modelos de carreira misteriosos, devido ao isolamento eà falta de aconselhamento, muitas se sentem "estagnadas", sem saber como direcionar suas apostas profissionais; sistemas de riscos e recompensas nos quais as mulheres se sentem desamparadas e, por isso, tendem a temer assumir riscos; e elevada pressão profissional, devido à cultura de horários muito intensivos (Hewlett et al., 2008).

Carreiras (2004) estudou amplamente a integração de mulheres nas forças armadas portuguesas, detectando as resistências e a manutenção das estruturas organizacionais da organização militar, não obstante a entrada de mulheres. Esses estudos e suas conclusões evocam as constatações da obra seminal Men and women of the corporation, de Rosabeth Moss Kanter (1993), embora esta apenas considerasse um fator explicativo da diferença entre mulheres e homens as estruturas organizacionais (as oportunidades de promoção, poder e recursos organizacionais e a proporção numérica de homens e mulheres em posições hierárquicas) e descurasse as concepções de gênero dominantes e estereotipadas. Ainda, seu trabalho foi fundamental no domínio da gestão por afastar as características individuais das mulheres como fatores explicativos da discriminação e exclusão.

Uma das componentes mais estudadas e disseminadas de sua teoria é a que equaciona a desproporção numérica de homens e mulheres em um mesmo contexto, entendendo que tal 
desproporção penaliza o grupo minoritário. Membros do grupo minoritário, as mulheres, no caso de ambientes masculinizados, são vistas como tokens, representantes dessa categoria, e não como indivíduos independentes, o que exacerba a estereotipia.

Essas abordagens seminais nos estudos de gênero e organizacionais são desafiadas pela introdução da perspectiva interseccional ao rejeitar a concepção de um sujeito unitário e homogêneo e introduzir a relação entre identidade e poder. Crenshaw (1989) a apresenta como lente que permite ver onde está o poder e onde convergem os eixos de desigualdade. Lembremos que a autora desenvolveu o conceito, na década de 1980, a partir do caso de uma mulher negra que havia sido discriminada no acesso ao emprego. A queixa dessa mulher não foi atendida pelo tribunal, pois a empresa alegou que contratava negros e mulheres. Entretanto, de fato, todos os negros recrutados pela empresa eram homens (operários na fábrica) e todas as mulheres eram brancas (administrativas na sede). Tal abordagem tem inspirado novas pesquisas e conceitualizações que interpretam a posição das mulheres nas organizações não apenas em termos de exclusão decorrente da norma masculina dominante, mas verificando a forma como as mulheres e feminilidades reconfiguradas estão a ser incluídas nos locais de trabalho contemporâneos (Lewis, 2014). Também vale referir o trabalho de Calás e Smircich (2011), que problematizam sob uma perspectiva feminista pós-estruturalista e pós-colonial, abordando a genderização em uma ótica intercional e estudando as subjetividades, as posições do sujeito e as instituições como processos temporais e espaciais que são produto e produtores de processos transnacionais. Em sua perspectiva, é a intersecção do sexo com raça, etnia, classe, sexualidades e outras subjetividades que possibilita compreender cabalmente os processos globais de desigualdade. Não negando sua importância heurística, a abordagem nos parece de menor utilidade nesta pesquisa centrada na estereotipia de gênero e em sua manifestação nas crenças e atitudes dos trabalhadores do sexo masculino inquiridos (de diversos grupos socioeconômicos e níveis de escolaridade, é certo).

\section{0 PAPEL SOCIAL DO GÊNERO}

As concepções de gênero, que determinam "quem faz o quê" com base no "como são as mulheres" e "como são os homens", são normativas e impõem-se na definição de capacidades, papéis e funções diferentes entre mulheres e homens (Monteiro, 2005). Como referiu Acker (1992), tem-se postulado a ideia do "trabalhador ideal", que trabalha o tempo inteiro e continuamente, pressupondo-se que tem o apoio de retaguarda de uma parceira, que assume o trabalho familiar (Lewis \& Cooper, 1995), em clara influência do modelo familiar de tipo parsoniano, no qual o homem é o "provedor do lar" e a mulher é a "cuidadora". A força dessa estereotipia que condiciona papéis sociais de gênero no trabalho, na família, na vida política e cívica está presente quer nas cognições dos indivíduos (homens e mulheres), quer nos modelos de funcionamento e gestão das organizações de trabalho. Segundo Aboim (2007) e Wall, Aboim e Cunha (2010), persiste no discurso uma política pró-natalista e familialista conservadora, de reenvio das mulheres para a casa e a domesticidade. No que diz respeito à posição social das mulheres e aos respectivos papéis, as atitudes se revelam mais conservadoras, sobressaindo uma oposição entre um ideal de mulher mãe e dona-de-casa e o ideal de mulher independente (Aboim, 2007, 2010).

Em relação às atitudes e aos valores, as autoras referem que o tradicional modelo de ganha-pão masculino tem perdido força em Portugal mais do que em outros países, pela predominância do modelo de duplo salário. Aboim (2010, p. 64), afirma que "os homens afastam-se do tradicional papel 
de homem provedor e autoritário, habitualmente ausente do quotidiano doméstico e parental, elogiando a participação, a presença e a cooperação masculina na família”. Em Portugal, em paralelo às elevadas taxas de atividade masculina, cresceram acentuadamente as taxas de atividade e de emprego das mulheres desde finais da década de 1960, atenuando o gap numérico entre homens e mulheres no mercado de trabalho. Essa evolução acarretou transformação da própria organização familiar, tendo a predominância do modelo de ganha-pão masculino do passado dado lugar ao do duplo emprego. Tal reorganização dos papéis de homens e mulheres na família veio desafiar o arquétipo tradicional da masculinidade, destacando-se hoje a disseminação de uma masculinidade cuidadora, já claramente menos estruturada em função do papel de provedor (Wall et al., 2010). Existem em Portugal fortes evidências que atestam a falta de sensibilidade social e o reduzido reconhecimento das desigualdades com base no sexo, e até certa desconsideração das conquistas legislativas e essas "novas" ideias e afirmações acerca da masculinidade (Ferreira et al., 2007).

Outros estudos dão conta de riscos psicossociais acrescidos para as mulheres em ambientes profissionais masculizados (Bergman, 2003), maior exposição a estresse e a assédio sexual (Parker \& Griffin, 2002), dificuldades de progressão na carreira (Crawford \& Unger, 2004), exclusão das redes de informação, desconsideração das opiniões e ideias das mulheres nas reuniões (Janz \& Pyke, 2000).

A realização de diagnósticos sensíveis às relações sociais de gênero é, pois, fundamental como instrumento da visibilização das assimetrias e intervenção para a mudança. Os diagnósticos e estudos devem munir os agentes da gestão e da decisão com os indicadores estruturais que traduzam a presença e manifestação das desigualdades e discriminação em função do sexo (segregação horizontal e vertical, por exemplo), bem como das principais dificuldades de conciliação e de utilização de direitos das pessoas da organização; os valores e as representações de gênero das pessoas ligadas à organização, as concepções de papéis sexuais e os estereótipos que determinam suas experiências e constrangem suas cognições, opções e atividades (Monteiro \& Ferreira, 2013)

Para conhecer as atitudes e concepções de gênero, investigações têm fornecido instrumentos para medir formas mais manifestas (Spence, Helmreich, \& Stapp, 1975) ou mais latentes de sexismo (Swim \& Cohen, 1997), bem como modelos de intervenções para combatê-lo (Zawadzki, Shields, Danube, \& Swim, 2014).

Swim e Hyers (2009, p. 407) definem sexismo como "atitudes, crenças e comportamentos individuais, bem como práticas organizacionais, institucionais e culturais que refletem avaliações negativas dos indivíduos com base no seu sexo e favorecem diferenças de estatuto de homens e mulheres". A Escala sobre Sexismo Moderno (Modern Sexism Scale - MS), de Swim e Cohen (1997), por exemplo, mede formas encobertas e sutis de sexismo. Essa medida constituiu complemento a um instrumento já da década de 1970, a Escala de Atitudes Face às Mulheres (Attitudes Toward Women Scale - AWS), que media formas explícitas de sexismo (Spence et al., 1975).

Em Portugal, tem-se estudado a evolução das atitudes de gênero, dando conta de uma ambivalência, já que se pende entre os dois polos de valores, um modernista e individualizante e outro tradicionalista e familialista (Aboim, 2007, 2010; Monteiro, Agostinho, \& Daniel, 2015; Torres, Coelho, Jerónimo, \& Cabrita, 2009). Segundo conclusões do projeto "Trabalhar e Cuidar na Europa", e em matéria de papéis de gênero, Portugal, apesar de encontrar afinidades com países de natureza mais "tradicionalista", situa-se no tipo "intermédio" (Torres et al., 2009). 
Tem havido evolução no modelo familiar de duplo salário e destituição do modelo de ganha-pão masculino, porém, no que se refere à posição social das mulheres e seus papéis ligados à domesticidade e maternidade, ainda se manifesta certo conservadorismo (Aboim, 2010). O declínio do modelo de ganha-pão masculino acarreta mudanças do lugar social das mulheres e também o faz relativamente ao modelo de masculinidade, reconfigurando as identidades e os papéis sociais dos homens nas diversas esferas de vida; por esse motivo, os próprios homens têm sido objeto de análise e potenciais agentes de mudança (Hearn et al., 2002).

\section{PROCEDIMENTOS METODOLÓGICOS}

O ponto de partida para esta pesquisa surgiu na sequência de um estudo elaborado na Suécia, no qual foi possível desenvolver uma nova medida de igualdade de gênero em locais de trabalho majoritariamente masculinos, possibilitando análises quantitativas das crenças masculinas sobre a desigualdade de gênero no trabalho.

Partindo da constatação de que não existia pesquisa suficiente acerca das opiniões dos homens sobre as razões para as desigualdades em nível organizacional e de que poderia ser relevante criar um inventário para medir as atitudes dos homens acerca da igualdade de gênero no trabalho, Bergman et al. (2014) desenvolveram o questionário “Men’s Polarized Gender Thinking Questionnaire (MPGQ)", adotado nesta pesquisa. O estudo teve início com uma pesquisa qualitativa, com entrevistas, realizada em 3 ambientes de trabalho masculinizados (Bergman, 2008), onde foi criada a categoria "pensamento polarizado de gênero", que descreve a coexistência de sentimentos e práticas opostos e paradoxais, em uma polarização entre perspectivas positivas e negativas. Ou seja, se por um lado emergiam expectativas de que as mulheres devem ajustar-se "às regras masculinas" existentes, por outro também se antevia a igualdade de gênero como uma realidade possível nas práticas futuras da organização. Isso conduziu à criação de 2 categorias analíticas: Igualdade de gênero ilusória (Illusory gender equality) e Visualização e prática da igualdade de gênero (Visualizing and practicing gender equality). A partir da construção do questionário e de vários procedimentos de validação, chegou-se a uma escala com 23 itens e um modelo explicativo composto por 6 fatores: a) Visões diferentes sobre o sucesso; b) Papéis estereotipados de gênero; c) Sexismo benevolente; d) Consciência da ordem de gênero; e) Consciência do sistema de normas masculinas; e f) Estratégias para a igualdade de gênero.

Os procedimentos de obtenção da versão portuguesa do MPGQ, cujo resultado é apresentado aqui, assentaram-se no método translate - translate back (Hill \& Hill, 2002). As versões em língua portuguesa e língua inglesa apresentaram elevado grau de semelhança, confirmando-se a equivalência entre a versão original e a traduzida (Hambleton, Merenda, \& Spielberger, 2005; International Test Commission, 2017). Em uma segunda fase, recorremos a especialistas em questões de gênero que eram simultaneamente investigadores fluentes na língua inglesa; analisando o conteúdo do questionário, validou-se a versão final.

Foi igualmente construído um pequeno questionário sociodemográfico para ser aplicado aos trabalhadores do sexo masculino de duas organizações. O estudo foi realizado em uma empresa municipal no setor do fornecimento e tratamento de águas (para preservar a anonimidade, designamos a empresa "Águas Limpas", que tem um efetivo de 270 pessoas, $75 \%$ das quais são homens) e em uma entidade pública no setor de emergência médica, da região centro (que designamos "SOS”, 
com 250 pessoas em serviço, $67 \%$ das quais são homens). A amostra totalizou 160 trabalhadores homens, que correspondem a 30,7\% do universo total de ambas as organizações. Em termos etários, a maior concentração de homens corresponde ao grupo etário dos 30 aos 39 anos (46,9\%); 49,9\% dos trabalhadores têm um nível de escolaridade correspondente ao $12^{\circ}$ ano. A grande maioria dos homens $(69,4 \%)$ é casada ou vive em união estável. No que s e refere ao tempo de serviço na organização, $37 \%$ dos trabalhadores estão a serviço de suas respectivas organizações por períodos que variam de 5 a 9 anos.

Os respontendentes eram instruídos a responder entre os pontos "completamente desacordo" (ponto mais baixo da escala) e "completamente de acordo" (o ponto mais elevado da escala). A aplicação dos questionários foi efetuada na organização Águas Limpas em junho e julho de 2016, enquanto na SOS a mesma aplicação ocorreu em novembro de 2016. A participação foi voluntária e assegurou-se a confidencialidade e o anonimato dos dados.

Aplicados e recolhidos os questionários, para o tratamento dos dados se utilizou o software SPSS, versão 21.0.

\subsection{Análise estatística}

Iniciamos o estudo da escala observando a consistência interna (alfa de Cronbach) dos itens que compõem o MPGQ. Em seguida, efetuou-se uma análise fatorial, para explorar a estrutura subjacente aos itens que compõem a subescala. O principal objetivo dessa análise é reduzir a complexidade das inter-relações entre as 23 variáveis observadas em um número relativamente pequeno de combinações lineares com tais variáveis. Em um primeiro momento, averiguou-se a adequação dos dados a esse tipo de procedimento estatístico, considerando-se o tamanho da amostra e a força da relação entre os itens. Para determinar o número de componentes principais a reter, observou-se tanto o critério de Kaiser (valor próprio - eingenvalue - igual ou superior a 1) como o de Cattell (distribuição gráfica scree plot-dos valores próprios). A aplicação desses critérios teve por objetivo obter uma indicação do número mínimo de fatores latentes a reter que fosse capaz de resumir apropriadamente a informação e que explicasse, consequentemente, uma proporção considerável da variância total.

$\mathrm{Na}$ descrição univariada das variáveis, recorremos a medidas de tendência central (média e mediana) e dispersão (desvio padrão). Na análise das diferenças entre a pontuação dos itens da escala e as variáveis sociodemográficas e profissionais, utilizou-se o teste U de Mann-Whitney (teste não paramétrico que compara as funções de distribuição de 1 variável de nível de mensuração pelo menos ordinal em 2 amostras independentes) e o $\mathrm{H}$ de Kruskal-Wallis (teste que compara as distribuições de 2 ou mais variáveis pelo menos ordinais observadas em 2 ou mais amostras independentes). Considerou-se o nível de significância $p \leq 0,05$ como indicativo da existência de diferenças estatisticamente significativas entre as médias das ordens dos grupos.

\section{RESULTADOS}

O cálculo do alfa de Cronbach revelou que o questionário MPGQ apresenta adequada consistência interna $(\alpha=0,77)$, respeitando os valores recomendados (Peterson, 1994; Daniel, Gomes \& Ferreira, 2015).

Em seguida, procedeu-se a uma análise fatorial exploratória para analisar a estrutura dimensional do MPGQ. Neste estudo, a indicação do uso do modelo fatorial na análise da MPGQ 
foi reforçada por um KMO de 0,81 (quanto mais próximo de 1, maior a adequação de uma análise fatorial) e por um teste de Bartlett com um nível de significância inferior a 0,001. Este último leva à rejeição da hipótese da matriz das correlações na população ser a matriz de identidade, mostrando que a correlação existente se dá entre as variáveis (Pestana \& Gageiro, 2008). Com o objetivo de verificar se os itens da MPGQ saturavam os fatores descritos na escala original, procedeu-se a uma análise fatorial exploratória por meio do método de componentes principais, para saber quais são as contribuições de cada item para cada fator. A análise fatorial exploratória permitiu, tal como na escala original, obter 6 fatores com valores superiores a 1 que explicam cumulativamente $61 \%$ da variância. Contudo, os resultados obtidos não foram idênticos aos do estudo da escala original. Não houve concordância dos itens pertencentes aos fatores e 7 itens saturam em mais do que 1 fator (crossloadings).

Diante dos resultados, partimos para a análise item a item. Para verificar se existiam diferenças entre as pontuações dos diferentes itens da escala, consoante as habilitações da literatura, utilizamos o teste $\mathrm{H}$ de Kruskal-Wallis. As variáveis que apresentavam significância no nível de $p<0,05$ constam na Tabela 1 . Assim, podemos constatar que as variáveis "A carreira profissional é mais importante para os homens do que para as mulheres" e "Para os homens é mais importante sustentar a família do que o papel parental" (Different views on sucess) apresentam diferenças estatisticamente significativas consoante as habilitações da literatura $\left(\mathrm{x}^{2}(4, n=155)=10,263\right.$, $p=0,036, \mathrm{x}^{2}(4, n=155)=10,878, p=0,028$, respectivamente). Nessas variáveis, o grupo com menor nível de habilitações da literatura apresenta maior mediana e média ( $M d=2$ e $M=2,46$, $M d=3$ e $M=2,77$, respectivamente).

As variáveis "As mulheres hesitam mais em expor as suas potencialidades (competências) do que os homens", "Quando se trata de negociar os salários, os homens são mais fortes a exigir do que as mulheres" e "Os homens normalmente conversam de forma mais brusca quando não há nenhuma mulher presente" também apresentaram significância no nível de $p<0,05\left(\mathrm{x}^{2}(4, n=155)=15,904\right.$, $p=0,003, \mathrm{x}^{2}(4, n=155)=15,598, p=0,004, \mathrm{x}^{2}(4, n=155)=25,012, p<0,001$, respectivamente $)$ e são as pessoas com menores habilitações escolares que apresentam as pontuações mais elevadas ( $M d=3$ e $M=2,77, M d=3$ e $M=3,38 M d=3$ e $M=3,15$, respectivamente).

No que concerne à variável "As mulheres em cargos de chefia dão mais apoio aos seus trabalhadores do que os homens em cargos de chefia", verificamos que são os homens que têm o $9^{\circ}$ ano de escolariedade que apresentam a pontuação mais elevada no nível da tendência central $(M d=3 \mathrm{e}$ $\left.M=2,75) \mathrm{x}^{2}(4, n=155)=18,943, p=0,01\right)$. Na variável "As pessoas, em geral, prestam mais atenção ao que os homens dizem" se constata que é o grupo com menor nível de habilitação que apresenta pontuação mais elevada $(M d=3$ e $\left.M=2,62) \mathrm{x}^{2}(4, n=155)=12,577, p=0,014\right)$. Já no que diz respeito à variável "Geralmente são atribuídas às mulheres tarefas diferentes das dos homens" são os grupos com menor nível de habilitações que apresentam pontuações mais elevadas e a diferença é de apenas 6 décimos, com vantagem para o grupo com o $9^{\circ}$ ano $(M d=3$ e $M=3,06) \mathrm{x}^{2}(4, n=155)=19,788$, $p=0,001)$. É o extremo superior das habilitações (mestrado) que apresenta a pontuação mais elevada, tanto na variável "A igualdade entre os homens e as mulheres no local de trabalho está ainda muito longe de ser alcançada" como na variável "Para aumentar o número de mulheres aqui na organização, isso só se conseguirá se a gestão/direção tomar medidas nesse sentido" ( $M d=3$ e $M=3,00) \mathrm{x}^{2}(4$, $n=155)=9,701, p=0,046$ e $(M d=3$ e $\left.M=2,80) \mathrm{x}^{2}(4, n=155)=19,265, p=0,001\right)$. 


\section{TABELA 1 MEDIDAS DE TENDÊNCIA CENTRAL E DISPERSÃO (M, MD E DP) E VALORES DE SIGNIFICÂNCIA DOS ITENS DO MPGQ CONSOANTE AS HABILITAÇÕES}

\begin{tabular}{|c|c|c|c|c|c|c|}
\hline & $<9^{\circ}$ & $9^{\circ}$ & $12^{\circ}$ & Lic. & Mest. & \\
\hline & $n=13$ & $n=16$ & $n=79$ & $n=42$ & $n=5$ & $P$ \\
\hline I- Visões diferentes acerca do sucesso & $\begin{array}{l}M \pm D P \\
(M d)\end{array}$ & $\begin{array}{l}M \pm D P \\
(M d)\end{array}$ & $\begin{array}{l}M \pm D P \\
(M d)\end{array}$ & $\begin{array}{l}M \pm D P \\
(M d)\end{array}$ & $\begin{array}{l}M \pm D P \\
(M d)\end{array}$ & \\
\hline $\begin{array}{l}\text { A carreira profissional é mais importante para } \\
\text { os homens do que para as mulheres }\end{array}$ & $\begin{array}{c}2,46 \pm 1,13 \\
\text { (2) }\end{array}$ & $\begin{array}{c}1,69 \pm 1,01 \\
\text { (1) }\end{array}$ & $\begin{array}{c}1,65 \pm 0,79 \\
\text { (1) }\end{array}$ & $\begin{array}{l}1,45 \pm 0,55 \\
\text { (1) }\end{array}$ & $\begin{array}{l}1,40 \pm 0,55 \\
\text { (1) }\end{array}$ & 0,036 \\
\hline $\begin{array}{l}\text { Para os homens é mais importante sustentar a } \\
\text { família do que o papel parental }\end{array}$ & $\begin{array}{c}2,77 \pm 0,60 \\
\text { (3) }\end{array}$ & $\begin{array}{c}2,44 \pm 0,81 \\
(2,5)\end{array}$ & $\begin{array}{c}2,24 \pm 0,82 \\
(2)\end{array}$ & $\begin{array}{l}2,07 \pm 0,75 \\
(2)\end{array}$ & $\begin{array}{c}1,80 \pm 0,84 \\
\text { (2) }\end{array}$ & 0,028 \\
\hline \multicolumn{7}{|l|}{ II - Papéis estereotipados de gênero } \\
\hline $\begin{array}{l}\text { As mulheres hesitam mais em expor as suas } \\
\text { potencialidades (competências) do que os } \\
\text { homens }\end{array}$ & $\begin{array}{c}2,77 \pm 0,44 \\
\text { (3) }\end{array}$ & $\begin{array}{c}2,31 \pm 0,70 \\
\text { (2) }\end{array}$ & $\begin{array}{l}2,03 \pm 0,68 \\
\text { (2) }\end{array}$ & $\begin{array}{c}2,10 \pm 0,66 \\
\text { (2) }\end{array}$ & $\begin{array}{c}2,20 \pm 0,45 \\
\text { (2) }\end{array}$ & 0,003 \\
\hline $\begin{array}{l}\text { Quando se trata de negociar os salários, os } \\
\text { homens são mais fortes a exigir do que as } \\
\text { mulheres }\end{array}$ & $\begin{array}{c}3,38 \pm 0,51 \\
\text { (3) }\end{array}$ & $\begin{array}{c}3,13 \pm 0,72 \\
\text { (3) }\end{array}$ & $\begin{array}{c}2,71 \pm 0,80 \\
(3)\end{array}$ & $\begin{array}{c}2,64 \pm 0,82 \\
\text { (3) }\end{array}$ & $\begin{array}{c}3,20 \pm 0,45 \\
\text { (3) }\end{array}$ & 0,004 \\
\hline $\begin{array}{l}\text { Os homens normalmente conversam de } \\
\text { forma mais brusca quando não há nenhuma } \\
\text { mulher presente }\end{array}$ & $\begin{array}{c}3,15 \pm 0,56 \\
(3)\end{array}$ & $\begin{array}{c}2,63 \pm 0,89 \\
(2,5)\end{array}$ & $\begin{array}{c}2,05 \pm 0,80 \\
(2)\end{array}$ & $\begin{array}{c}2,14 \pm 0,57 \\
(2)\end{array}$ & $\begin{array}{c}2,20 \pm 0,84 \\
(2)\end{array}$ & $<0,001$ \\
\hline \multicolumn{7}{|l|}{ III - Sexismo benevolente } \\
\hline $\begin{array}{l}\text { As mulheres em cargos de chefia dão mais } \\
\text { apoio aos seus trabalhadores do que os } \\
\text { homens em cargos de chefia }\end{array}$ & $\begin{array}{c}1,83 \pm 0,39 \\
\text { (2) }\end{array}$ & $\begin{array}{c}2,75 \pm 0,68 \\
\text { (3) }\end{array}$ & $\begin{array}{c}2,01 \pm 0,64 \\
\text { (2) }\end{array}$ & $\begin{array}{c}2,00 \pm 0,54 \\
\text { (2) }\end{array}$ & $\begin{array}{l}1,80 \pm 0,45 \\
\text { (2) }\end{array}$ & 0,001 \\
\hline \multicolumn{7}{|l|}{ IV - Consciência da ordem de gênero } \\
\hline $\begin{array}{l}\text { As pessoas, em geral, prestam mais atenção } \\
\text { ao que os homens dizem }\end{array}$ & $\begin{array}{c}2,62 \pm 0,77 \\
\text { (3) }\end{array}$ & $\begin{array}{c}2,25 \pm 0,78 \\
\text { (2) }\end{array}$ & $\begin{array}{l}2,19 \pm 0,74 \\
\text { (2) }\end{array}$ & $\begin{array}{c}1,86 \pm 0,61 \\
\text { (2) }\end{array}$ & $\begin{array}{c}2,20 \pm 0,84 \\
\text { (2) }\end{array}$ & 0,014 \\
\hline $\begin{array}{l}\text { Geralmente são atribuídas às mulheres } \\
\text { tarefas diferentes das dos homens }\end{array}$ & $\begin{array}{c}3,00 \pm 0,58 \\
\text { (3) }\end{array}$ & $\begin{array}{c}3,06 \pm 0,44 \\
\text { (3) }\end{array}$ & $\begin{array}{c}2,51 \pm 0,78 \\
(3)\end{array}$ & $\begin{array}{c}2,62 \pm 0,70 \\
\text { (3) }\end{array}$ & $\begin{array}{c}1,60 \pm 0,55 \\
\text { (3) }\end{array}$ & 0,001 \\
\hline \multicolumn{7}{|l|}{$\begin{array}{l}\text { V - Consciência do sistema de normas } \\
\text { masculinas }\end{array}$} \\
\hline $\begin{array}{l}\text { A igualdade entre os homens e as mulheres } \\
\text { no local de trabalho está ainda muito longe } \\
\text { de ser alcançada }\end{array}$ & $\begin{array}{c}2,54 \pm 1,05 \\
\text { (3) }\end{array}$ & $\begin{array}{c}2,69 \pm 0,60 \\
\text { (3) }\end{array}$ & $\begin{array}{c}2,24 \pm 0,84 \\
(2)\end{array}$ & $\begin{array}{c}2,24 \pm 0,79 \\
(2)\end{array}$ & $\begin{array}{c}3,00 \pm 0,71 \\
\text { (3) }\end{array}$ & 0,046 \\
\hline \multicolumn{7}{|l|}{ VI - Estratégias para a igualdade de gênero } \\
\hline $\begin{array}{l}\text { Para aumentar o número de mulheres aqui } \\
\text { na organização, isso só se conseguirá se a } \\
\text { gestão/direção tomar medidas nesse sentido }\end{array}$ & $\begin{array}{c}2,62 \pm 0,77 \\
\text { (3) }\end{array}$ & $\begin{array}{c}2,69 \pm 0,80 \\
(3)\end{array}$ & $\begin{array}{c}1,96 \pm 0,78 \\
(2)\end{array}$ & $\begin{array}{c}2,17 \pm 0,73 \\
(2)\end{array}$ & $\begin{array}{c}2,80 \pm 0,84 \\
\text { (3) }\end{array}$ & 0,001 \\
\hline
\end{tabular}

Fonte: Elaborado pelos autores. 
Para verificar se existiam diferenças entre as pontuações dos diferentes itens da escala consoante as instituições onde trabalham, utilizamos o teste $\mathrm{U}$ de Mann-Whitney. As variáveis que apresentavam significância no nível de $p<0,05$ constam na Tabela 2. Assim, podemos constatar que variáveis "A carreira profissional é mais importante para os homens do que para as mulheres" e "Os homens consideram mais do que as mulheres que o salário é uma importante medida do seu sucesso profissional" (Different views on sucess) apresentam diferenças estatisticamente significativas consoante as instituições onde trabalham $(U=2272,000, \mathrm{z}=-2,834, p=0,005, U=7501,500, \mathrm{z}=-2,039, p=0,041$, respectivamente). Os funcionários das Águas Limpas apresentam pontuações mais elevadas no nível da média $(M d=2 \mathrm{e}$ $M=1,90, M d=2$ e $M=2,32$, respectivamente). As três variáveis "comprometidas" com a esterotipia de gênero "As mulheres hesitam mais em expor as suas potencialidades (competências) do que os homens", "Os homens normalmente conversam de forma mais brusca quando não há nenhuma mulher presente" e "Quando se trata de negociar os salários, os homens são mais fortes a exigir do que as mulheres" apresentam igualmente pontuações mais elevadas na empresa Águas Limpas ( $M d=2$ e $M=2,32$, $M d=3$ e $M=2,60, M d=3$ e $M=2,60),(U=2306,500, \mathrm{z}=-2,714, p=0,007, U=2312,000, \mathrm{z}=-2,733$, $p=0,006$, respectivamente). As duas últimas variáveis que apresentaram significância estatística são "Para aumentar o número de mulheres aqui na organização, isso só se conseguirá se a gestão/direção tomar medidas nesse sentido" e "É mais importante dar um apoio mais ativo às mulheres em cargos de chefia do que aos homens em cargos de chefia" ( $U=1770,500, \mathrm{z}=-4,544, p<0,001, U=2286,000$, $\mathrm{z}=-2,661, p=0,008)$, ambas apresentam pontuações médias superiores na Águas Limpas $(M d=3 \mathrm{e}$ $M=2,55, M d=2$ e $M=2,58$, respectivamente).

\section{TABELA 2 MEDIDAS DE TENDÊNCIA CENTRAL E DISPERSÃO (M, MD E DP) E VALORES DE SIGNIFICÂNCIA DOS ITENS DO MPGQ CONSOANTE A INSTITUIÇÃO DE TRABALHO}

\begin{tabular}{|c|c|c|c|}
\hline & $\begin{array}{l}\text { Águas livres } \\
\quad n=42\end{array}$ & $\begin{array}{l}\text { SOS } \\
n=5\end{array}$ & $P$ \\
\hline I - Visões diferentes acerca do sucesso & $\begin{array}{l}M \pm D P \\
(M d)\end{array}$ & $\begin{array}{c}M \pm D P \\
(M d)\end{array}$ & \\
\hline $\begin{array}{l}\text { A carreira profissional é mais importante para os homens do que } \\
\text { para as mulheres }\end{array}$ & $\begin{array}{l}1,90 \pm 0,90 \\
(2)\end{array}$ & $\begin{array}{l}1,52 \pm 0,72 \\
(1)\end{array}$ & 0,005 \\
\hline $\begin{array}{l}\text { Os homens consideram mais do que as mulheres que o salário é } \\
\text { uma importante medida do seu sucesso profissional }\end{array}$ & $\begin{array}{l}2,32 \pm 0,98 \\
(2)\end{array}$ & $\begin{array}{l}1,99 \pm 0,84 \\
(2)\end{array}$ & 0,041 \\
\hline \multicolumn{4}{|l|}{ II - Papéis estereotipados de gênero } \\
\hline $\begin{array}{l}\text { As mulheres hesitam mais em expor as suas potencialidades } \\
\text { (competências) do que os homens }\end{array}$ & $\begin{array}{l}2,32 \pm 0,65 \\
(2)\end{array}$ & $\begin{array}{l}2,03 \pm 0,67 \\
(2)\end{array}$ & 0,007 \\
\hline $\begin{array}{l}\text { Os homens normalmente conversam de forma mais brusca quando } \\
\text { não há nenhuma mulher presente }\end{array}$ & $\begin{array}{l}3,02 \pm 0,75 \\
\text { (3) }\end{array}$ & $\begin{array}{l}2,68 \pm 0,79 \\
\text { (3) }\end{array}$ & $<0,001$ \\
\hline $\begin{array}{l}\text { Quando se trata de negociar os salários, os homens são mais fortes } \\
\text { a exigir do que as mulheres }\end{array}$ & $\begin{array}{l}2,60 \pm 0,81 \\
\text { (3) }\end{array}$ & $\begin{array}{l}2,01 \pm 0,70 \\
(2)\end{array}$ & 0,006 \\
\hline
\end{tabular}




\begin{tabular}{|c|c|c|c|}
\hline VI - Estratégias para a igualdade de gênero & $\begin{array}{c}\text { Águas livres } \\
n=42\end{array}$ & $\begin{array}{l}\text { sOS } \\
n=5\end{array}$ & $P$ \\
\hline $\begin{array}{l}\text { Para aumentar o número de mulheres aqui na organização, isso só } \\
\text { se conseguirá se a gestão/direção tomar medidas nesse sentido }\end{array}$ & $\begin{array}{l}2,55 \pm 0,77 \\
\text { (3) }\end{array}$ & $\begin{array}{c}1,94 \pm 0,75 \\
(2)\end{array}$ & $<0,001$ \\
\hline $\begin{array}{l}\text { É mais importante dar um apoio mais ativo às mulheres em cargos } \\
\text { de chefia do que aos homens em cargos de chefia }\end{array}$ & $\begin{array}{l}2,58 \pm 2,67 \\
(2)\end{array}$ & $\begin{array}{l}1,96 \pm 0,67 \\
\text { (2) }\end{array}$ & 0,008 \\
\hline
\end{tabular}

Fonte: Elaborado pelos autores.

\section{DISCUSSÃO/CONCLUSÕES}

$\mathrm{Na}$ análise dos dados estatísticos, constatou-se a existência de concepções informadas pelas representações sociais de gênero.

Com base nos resultados, pôde-se chegar às seguintes conclusões: Em relação às diferenças significativas encontradas no que se refere às habilitações da literatura, foi perceptível que a maior parte dos homens com baixo nível de escolarização concorda com os itens de caráter mais conservador. Esses itens revelam essencialmente que o papel do homem passa pelo modelo tradicional de gênero que atribui aos homens o papel de provedor do lar e às mulheres o papel de cuidar da família. Eles também revelam que os homens consideram que a profissão é mais importante para os homens do que para as mulheres.

Por outro lado, também se observa uma atitude de maior consciencialização das desigualdades ainda existentes nos locais de trabalho e da necessidade de intervenção específica por parte dos homens com maior nível de instrução, no que se refere aos seguintes itens: "Para aumentar o número de mulheres aqui na organização, isso só se conseguirá se a gestão/direção tomar medidas nesse sentido" e "A igualdade entre os homens e as mulheres no local de trabalho está ainda muito longe de ser alcançada".

Esses resultados demonstram que as atitudes dos homens em relação à igualdade de gênero não se distribuem aleatoriamente. As participações no mercado de trabalho dos homens com níveis de instrução mais elevados se articulam com atitudes mais igualitaristas. A escolarização e educação dos indivíduos são consideradas fundamentais, pois se destacam como importante vetor de aquisição de um sistema de crenças e de valores sociais mais igualitários sobre o tema da relação entre os sexos. Esses achados corroboram alertas lançadas pelas abordagens da interseccionalidade.

Tais dados vêm confirmar os achados de outras pesquisas relativamente à persistência de valores tradicionalistas de gênero. De fato, têm-se verificado valorização e ênfase da maternidade e do cuidado das crianças na identidade feminina, ao passo que aos homens se atribui maior valorização da atividade profissional. Essa constatação comprova a ambivalência e dicotomia de valores e atitudes, não obstante uma fuga à convencional polarização de papéis "masculinos" e "femininos" rígida e estereotipadamente definidos, também encontrada em outros estudos, em Portugal - reflexo da evolução para o modelo de "duplo salário" (Aboim, 2010).

Nas investigações sobre a produção das relações sociais de gênero, o homem só muito mais recentemente foi equacionado como objeto de estudo, ao se perceber que o modelo tradicional de masculinidade estava em transformação (Almeida, Ferreira, Ferrão, \& André, 1995; Amâncio, 1994; 
Brod \& Kaufman, 1994; Kimmel \& Messner, 1995) e que a tensão trabalho-família também se lhe aplicava (Crompton, 1999). Se a manutenção, na esfera da família, das desigualdades entre homens e mulheres é indubitável, é certo que também se evidencia um movimento de entrada do homem no universo da produção doméstica e parental (Almeida \& Wall, 2001; Perista, 2002; Torres, 2001; Wall, 2005).

O principal objetivo das políticas para a igualdade de gênero é alcançar a igualdade de oportunidades entre mulheres e homens, mas também de resultados. Segundo Sörlin, Ohman, Blomstedt, Stenlund, e Lindholm (2011), a proporção de trabalhadores/as é um indicador útil globalmente, sendo razoável supor que um pequeno número de mulheres ou de homens pode sentir desvantagem em uma organização.

Outra variável onde foram detectadas diferenças significativas foi a que diz respeito às duas instituições onde foi elaborada esta pesquisa. Novamente com o apoio da análise das médias, concluiuse que em contextos organizacionais altamente masculinizados a estereotipia de gênero tende a ser mais acentuada e que os trabalhadores da empresa Águas Limpas constituem a empresa mais masculinizada, onde as funções são mais estereotipadamente representadas como "masculinas", aqueles nos quais a expressão das diferenças assente nas relações sociais de gênero é superior em relação à organização menos segregada. Daqui emerge um contributo desta pesquisa, comprovando que medidas e políticas de combate à segregação horizontal apresentam potencial de alteração da estereotipia de gênero.

Este estudo permitiu identificar a perceção dos trabalhadores masculinos de duas organizações acerca das desigualdades de gênero. É importante expandir a aplicação e o teste do instrumento usado, de modo a aprimorar sua capacidade de medida no contexto português. De forma mais global, este trabalho constitui contributo no domínio dos estudos de gênero e organizacionais, fornecendo ferramentas analíticas para diagnósticos e pesquisas com enfoque quantitativo demonstrativas do fenômeno de genderização das organizações. 


\section{REFERÊNCIAS}

Aboim, S. (2007). Clivagens e continuidades de género face aos valores da vida familiar em Portugal e noutros países europeus. In K. Wall, \& Amâncio, L. (Eds.), Família e género em Portugal e na Europa (pp. 35-91). Lisboa, Portugal: Imprensa de Ciências Sociais.

Aboim, S.(2010). A vida familiar no masculino: negociando velhas e novas masculinidades. Lisboa, Portugal: Comissão para a Igualdade no Trabalho e no Emprego.

Acker, J. (1990) Hierarchies, jobs, bodies: A theory of gendered organizations. Gender and Society, 4(2), 139-158. Recuperado de http://www.jstor.org/ stable/189609

Acker, J. (1992). Gendering organizational theory. In A. J. Mills, \& P. Tancred (Eds.), Gendering organizational analysis (pp. 248-260). Newbury Park, CA: SAGE.

Acker, J. (2009). From glass ceiling to inequality regimes. Sociologie du Travail, 51(2), 199-217.

Agência Europeia para a Segurança e Saúde no Trabalho. (2013). Risks and trends in the safety and health of women at work research. Luxembourg City, Luxembourg: Publications Office of the European Union.

Almeida, A. M. N., Ferreira, C., Ferrão, F., \& André, I. M. (1995). Os padrões recentes da fecundidade em Portugal. Lisboa, Portugal: Comissão para a Igualdade e para os Direitos das Mulheres.

Almeida, A. M. N., \& Wall, K. (2001). Família e quotidiano: movimentos e sinais de mudança. In J. B. Brito (Org.), O país em revolução (pp. 277 307). Lisboa, Portugal: Ed. Notícias.

Amâncio, L. (1992). As assimetrias nas representações do género. Revista Crítica de Ciências Sociais, $34,9-22$.

Amâncio, L. (1994). Masculino e feminino: a construção social da diferença. Porto, Portugal: Afrontamento.

Benschop, Y., \& Verloo, M. (2006). Sisyphus' sisters: can gender mainstreaming escape the genderedness of organizations? Journal of Gender Studies, 15(1), 19-33.
Bergman, B. (2003). The validation of the women workplace culture questionnaire: gender-related stress and health for Swedish working women. Sex Roles, 49, 287-297.

Bergman, B. (2008). Polarized gender balance: visions or norms in male-dominated work places? International Journal of Qualitative Studies on Health and Well-Being, 3(3), 165-175.

Bergman, B., Larsman, P., \& Löve, J. (2014). Psychometric evaluation of the "Men's Polarized Gender Thinking Questionnaire (MPGQ)". Gender in Management: An International Journal, 29(4), 194-209.

Broadbridge, A., \& Hearn, J. (2008). Gender and management: new directions in research and continuing patterns in practice. British Journal of Management, 19(1), 38-49.

Broadbridge, A., \& Simpson, R. (2011). 25 years on: reflecting on the past and looking to the future in gender and management research. British Journal of Management, 22(3), 470-483.

Brod, H., \& Kaufman, M. (Eds.). (1994). Theorizing masculinities. Thousand Oaks, CA: SAGE.

Calás, M.B. \& Smircich, L. (2011). From the 'Woman's Point of View' Ten Yeas Late: Toward a Feminist Organization Studies. In S.R. Clegg, C. Hardy, T.B. Lawrence \& W.R. Nord (Eds.) The SAGE Handbook of Organization Sudies (pp. 284-346). London: SAGE Publications. Recuperado de https:// doi.org/10.4135/9781848608030.n9

Carreiras, H. (2004). Mulheres em contextos atípicos: lógicas de exclusão e estratégias de integração feminina nas forças armadas. Etnográfica, 8(1), 91-115.

Collinson, D. (1992). Managing the shopfloor: subjectivity, masculinity and workplace culture. Berlin, Germany: Walter de Gruyter.

Crawford, M., \& Unger, R. (2004). Women and gender. A feminist psychology. New York, NY: McGraw-Hill.

Crenshaw, K. (1989). Demarginalizing the intersection of race and sex: a black feminist critique of antidiscrimination doctrine, feminist theory and antiracist politics. University of Chicago Legal Forum, 1, 139-167. 
Crompton, R. (Ed.). (1999). Restructuring gender relations and employment: the decline of the male breadwinner. Oxford, England: Oxford University Press.

Daniel, F., Gomes, A. \& Ferreira, P.L. (2015). Contributo para a discussão da avaliação da fiabilidade de um instrument de medição. Revista de Enfermagem Referência, 4(7), 129-137.

Fagan, C., \& Burchell, B. (2002). Gender, jobs and working conditions in the European Union. Dublin, Ireland: European Foundation for the Improvement of Living and Working Conditions.

Fagenson, E. A. (1990). At the heart of women in management research: theoretical and methodological approaches and their biases. Journal of Business Ethics, 9(4-5), 267-274.

Fenstermaker, S., West, C., \& Zimmerman, D. H. (1991). Gender inequality: new conceptual terrain. In R. Lesser-Blumberg (Ed.), Gender, family and economy: the triple overlap, 289-307. California: SAGE Publications. Recuperado de https://doi. org/10.4135/9781483325415.n14

Ferreira, V., Silveirinha, M. J., Portugal, S., Vieira, C., Monteiro, R., Duarte, M., \& Lopes, M. (2007). Estudo de avaliação do II Plano Nacional para a Igualdade. Coimbra, Portugal: Centro de Estudos Sociais.

Hambleton, R. K., Merenda, P. F., \& Spielberger, C. D. (2005). Adapting educational and psychological tests for cross-cultural assessement. Mahwah, NJ: L. Erlbaum.

Hearn, J., Pringle, K., Müller, U., Oleksy, E., Lattu, E., Tallberg, T., \& Olsvik, E. (2002). Critical studies on men in ten European countries: (3) The state of law and policy. Men and Masculinities, 5(2), 192-217.

Hewlett, S. A., Luce, C. B., Servon, L. J., Sherbin, L., Shiller, P., Sosnovich, E., \& Sumberg, K. (2008). The Athena Factor: reversing the brain drain in science, engineering, and technology. New York, NY: Center for Work-Life Policy.

Hill, M., \& Hill, A. (2002). Investigação por questionário (2 ed.). Lisboa, Portugal: Sílabo.

International Test Commission. (2017). ITC guidelines for translating and adapting tests (2 ed.). Recuperado de https://www.intestcom.org/files/ guideline_test_adaptation_2ed.pdf
Janz, T. A., \& Pyke, S. W. (2000). A scale to assess student's perceptions of academic climates. The Canadian Journal of Higher Education, 30(1), 89-122.

Kanter, R. M. (1993). Men and women of the corporation. New York, NY: Basic Books.

Kimmel, M., \& Messner, M. (1995). Men's lives. Boston, MA: Allyn \& Bacon.

Lewis, P. (2014). Postfeminism, femininities and organization studies: exploring a new agenda. Organization Studies, 35(12), 1845-1866.

Lewis, S., \& Cooper, C. L. (1995). Balancing the work/home interface: a European perspective. Human Resource Management Review, 5(4), 289-305.

Lopes, M. (2009). Trabalho e parentalidade: um estudo sobre a acomodação e custos da maternidade e paternidade para os índividuos e as organizações (Dissertação de Mestrado). Coimbra, Portugal: Universidade de Coimbra.

Monteiro, R. (1995). Feminilidade na fábrica: a (re) construção das identidades sexuais nas organizações de trabalho (Dissertação de Licenciatura). Coimbra, Portugal: Universidade de Coimbra.

Monteiro, R. (2005). O que dizem as mães: mulheres trabalhadoras e suas experiências. Coimbra, Portugal: Quarteto.

Monteiro, R. (2010). A emergência do feminismo de Estado em Portugal: uma história da criação da Comissão da Condição Feminina. Lisboa, Portugal: CIG.

Monteiro, R., \& Ferreira, V. (2013). Planos para a igualdade de género nas organizações: contributo para o desenho e realização dos diagnósticos organizacionais. Sociedade e Trabalho, 44, 123-136.

Monteiro, R., Agostinho, L. \& Daniel, F. (2015). Um diagnóstico da desigualdade de gênero num município em Portugal: estruturas e represenações. Revista de Administração Pública, 49(2), 423-446. Recuperado de http://dx.doi.org/10.1590/0034-7612130497

Morgan, D. (1992). Discovering men: critical studies on men and masculinities. London, England: Routledge.

Nogueira, C. (2001). Feminismo e discurso do género na psicologia social. Psicologia \& Sociedade, 13(1), 107-128.

Ornelas, M. G. R., Matos, F. R. N., Machado, D. Q., \& Mesquita, R. F. (2017). Precariado e género: 
a trajectória de mulheres portuguesas rumo a uma nova classe em formação. Ámbitos: Revista Internacional de Comunicación, 39, 1-12.

Parker, S. K., \& Griffin, M. A. (2002). What is so bad about a little name-calling? Negative responses of gender harassment for over performance demands and distress. Journal of Occupational Health Psychology, 7(3), 195-210.

Pestana, M. \& Gageiro, J. (2008). Análise de dados para ciências sociais: A complementaridade do SPSS (2 ed.). Lisboa, Portugal: Edições Sílabo.

Peterson, R. (1994). A meta-analysis of Cronbach's coeficiente alpha. Journal of Consumer Research, 21(2), 381-391,

Perista, H. (2002). Manutenção das desigualdades de género nos usos do tempo dedicado às tarefas domésticas e aos filhos. Análise Social, 163, 447-474.

Reskin, B. F., \& Padavic, I. (1994). Women and men at work. Thousand Oaks, CA: Pine Forge Press.

Sealy, R., \& Singh, V. (2010). The importance of role models and demographic context for senior women's work identity development. International Journal of Management Reviews, 12, 284-300.

Smith, P., Crittenden, N., \& Caputi, P. (2012). Measuring women's beliefs about glass ceilings: development of the Career Pathway Survey. Gender in Management: An International Journal, 27(2), 68-80.

Sörlin, A., Ohman, A., Blomstedt, Y., Stenlund, H., \& Lindholm, L. (2011). Measuring the gender gap in organizations. International Journal, 26(4), 275-288.
Spence, J. T., Helmreich, R. L., \& Stapp, J. (1975). Ratings of self and peers on sex role attributes and their relation to self-esteem and conceptions of masculinity and femininity. Journal of Personality and Social Psychology, 32, 29-39.

Swim, J. K., \& Cohen, L. L. (1997). Overt, covert, and subtle sexism. A comparison between the attitudes toward women and modern sexism scales. Psychology of Woman Quarterly, 21(1), 103-118.

Swim, J. K., \& Hyers, L. L. (2009). Sexism. In T. D. Nelson (Ed.), Handbook of prejudice, stereotyping and discrimination (pp. 407-430). New York, NY: Psychology Press.

Torres, A. (2001). Sociologia do casamento. A família e a questão feminina. Oeiras, Portugal: Celta.

Torres, A., Coelho, B., Jerónimo, P., \& Cabrita, M. (2009, 26 de novembro). Workcare/trabalhar ecuidar na Europa. Recuperado de http://analiatorres.com/ pdf/synopsis_WORKCARE[2].pdf

Wall, K. (Org.). (2005). Famílias em Portugal. Percursos, interacções, redes sociais. Lisboa, Portugal: Imprensa de Ciências Sociais.

Wall, K., Aboim, S., \& Cunha, V. (2010). A vida familiar no masculino: negociando velhas e novas masculinidades. Lisboa, Portugal: Comissão para a Igualdade no Trabalho e no Emprego.

Zawadzki, M., Shields, S., Danube, C., \& Swim, J. (2014). Reducing the endorsement of sexism using experiential learning: the workshop activity for gender equity simulation (WAGES). Psychology of Women Quarterly, 38(1), 75-92. 


\section{Rosa Monteiro}

https://orcid.org/0000-0002-2429-5590

Doutora em sociologia; Professora da faculdade de Economia da Universidade de Coimbra; Investigadora do Centro de Estudos Sociais da Universidade de Coimbra. E-mail: monteiro.rosa14@gmail.com

\section{Amélia Filipa Soares Vieira}

https://orcid.org/0000-0002-6492-3037

Mestre em Gestão de Recursos Humanos e Comportamento Organizacional pelo Instituto Superior Miguel Torga. E-mail: filipavieira_01@hotmail.com

\section{Fernanda Daniel}

iD

https://orcid.org/0000-0002-2202-1123

Doutora em Psicologia e Serviço Social; Professora auxiliar do Instituto Superior Miguel Torga; Investigadora do Centro de Estudos e Investigação em Saúde da Universidade de Coimbra (CEISUC).

E-mail: fernanda-daniel@ismt.pt

\section{Alexandre Gomes da Silva}

https://orcid.org/0000-0001-5163-9670

Doutor em Estatística; Professor coordenador da Coimbra Business School/ISCAC; Investigador do Centro de Estudos e Investigação em Saúde da Universidade de Coimbra (CEISUC). E-mail: alexmfgs@gmail.com

\section{Fátima Regina Ney Matos}

https://orcid.org/0000-0002-2331-9335

Doutora em Administração; Professora auxiliar do Instituto Superior Miguel Torga. E-mail: fneymatos@ismt.pt 